\title{
The Advantages of Nanoparticles for PET
}

\author{
Michael J. Welch ${ }^{1}$, Craig J. Hawker ${ }^{2}$, and Karen L. Wooley ${ }^{3,4}$ \\ ${ }^{1}$ Departments of Radiology and Chemistry, Washington University, Saint Louis, Missouri; ${ }^{2}$ Materials Research Laboratory, Department \\ of Materials, and Department of Chemistry and Biochemistry, University of California Santa Barbara, Santa Barbara, California; \\ ${ }^{3}$ Departments of Chemistry and Radiology, Washington University, Saint Louis, Missouri; and ${ }^{4}$ Department of Chemistry, Texas A\&M \\ University, College Station, Texas
}

Nanoparticles have an advantage for molecular imaging in that many functionalities can be added to the surface and interior of the particle. This brief review focuses on the design of nanomaterials that take advantage of PET. An evolutionary approach is presented, leading to the optimization of the nanoparticle composition and structure to achieve controlled in vivo circulation and tissue-selective targeting. Organic and inorganic nanostructures are included. Nanoprobes for PET of angiogenesis and cancer are highlighted.

Key Words: positron emission tomography; PET; nanoparticles; nanoscale structures; multifunctionality

J Nucl Med 2009; 50:1743-1746

DOI: 10.2967/jnumed.109.061846

There is currently great interest in the application of nanoparticles for molecular imaging. This has led to the development of both organic and inorganic nanoparticles that have been functionalized so that radionuclides, targeting ligands, and polyethylene glycol can be attached to provide the imaging signal, target the particle, and alter the pharmacokinetics of the particle. The structure of the nanoparticle allows a broad range of radiolabeled chemistries to be used to attach various PET nuclides to the particle. Using nanoparticles with PET allows the quantitation of PET (1) and the multifunctionality of nanoparticles to be taken advantage of.

Nanoscopic materials fill a critical position between the macroscopic world and molecular-level detail and can be designed to offer unique advantages when compared with both macroscopic materials and molecular systems. As structures are built up from their constituent parts, the surface area-to-volume ratio decreases. For instance, for a spheric object, the surface area increases by a factor of $r^{2}$, whereas the volume increases cubically; the surface area-tovolume ratio, therefore, decreases with increasing sphere radius. Considered in the opposite sense, the volume-to-surface area ratio increases with increasing diameter, so that on going from small molecules or macromolecules and building up to nanoscale

Received Jun. 18, 2009; revision accepted Aug. 25, 2009.

For correspondence or reprints contact: Michael J. Welch, $510 \mathrm{~S}$. Kingshighway Blvd., Departments of Radiology and Chemistry, Washington University, Saint Louis, MO 63110.

E-mail: welchm@wustl.edu

COPYRIGHT @ 2009 by the Society of Nuclear Medicine, Inc. structures, an internal volume is created. This characteristic has generated significant interest in synthetic nanomaterials that can serve as vessels for the transport and delivery of imaging and therapeutic agents. Nanoscopic objects possess internal packaging capacity and a sufficient surface area for presentation of multiple types and numbers of active elements; that is, they can be designed to have an optimal balance of internal volume and external surface area.

The promising attributes of nanomaterials for targeted imaging include their ability to deliver large numbers of imaging agents per each targeted molecular recognition event to achieve high-sensitivity imaging and their ability to deliver several different types of those imaging agents to perform multimodality imaging. Several examples of synthetic nanomaterials that carry radiolabels for PET and biologically active ligands for targeting will be highlighted. The ability to perform highly sensitive imaging, by taking advantage of the power of PET and the capacity of the nanoscale framework, will be demonstrated.

\section{STATE OF THE ART: DESIGNS, IN VIVO BIODISTRIBUTION, AND PET}

The impact of molecular versus nanoscopic size was elegantly demonstrated by PET and biodistribution studies that compared a library of starlike polymer nanostructures and their linear-arm polymer precursors (Fig. 1) (2). Each linear-arm polymer comprised a methoxy-terminated poly(ethylene glycol) (mPEG) chain segment, for stealth behavior in avoiding rapid in vivo clearance by the mononuclear phagocytic system (MPS), and a segment that was functionalized with 1,4,7,10-tetraazacyclododecanetetraacetic acid (DOTA) units, for chelation of ${ }^{64} \mathrm{Cu}$. The chemical compositions and molecular sizes were well defined for a series of arm polymers using controlled radical polymerization techniques. Their $\omega$-chain termini remained active to allow for the direct conversion of the arms into star polymers by multimolecular cross-linking polymerization, bringing together 10-22 arms, depending on the conditions, by reaction with a bifunctional monomer. Radiolabeling with ${ }^{64} \mathrm{Cu}$ acetate, followed by purification, gave greater than $95 \%$ radiochemical purity, and the nanomaterials were stably labeled; they retained greater than $95 \%$ of the ${ }^{64} \mathrm{Cu}$ after $48 \mathrm{~h}$ incubation in rat serum at $36^{\circ} \mathrm{C}$ in vitro. The lineararm polymers having a hydrodynamic diameter $\left(D_{h}\right)$ of $4.6 \pm 0.3 \mathrm{~nm}$ were cleared rapidly from the blood through renal excretion and did not accumulate in other organs. In contrast, the nanoscale star polymer structures $\left(\mathrm{D}_{\mathrm{h}}\right.$ ranged from 25 to $70 \mathrm{~nm}$, depending on the structure) circulated for many hours, eventually being captured primarily in the liver and spleen. The dramatic difference in biodistribution is illustrated in the superimposed small-animal PET and micro-CT images of Figure 1. 


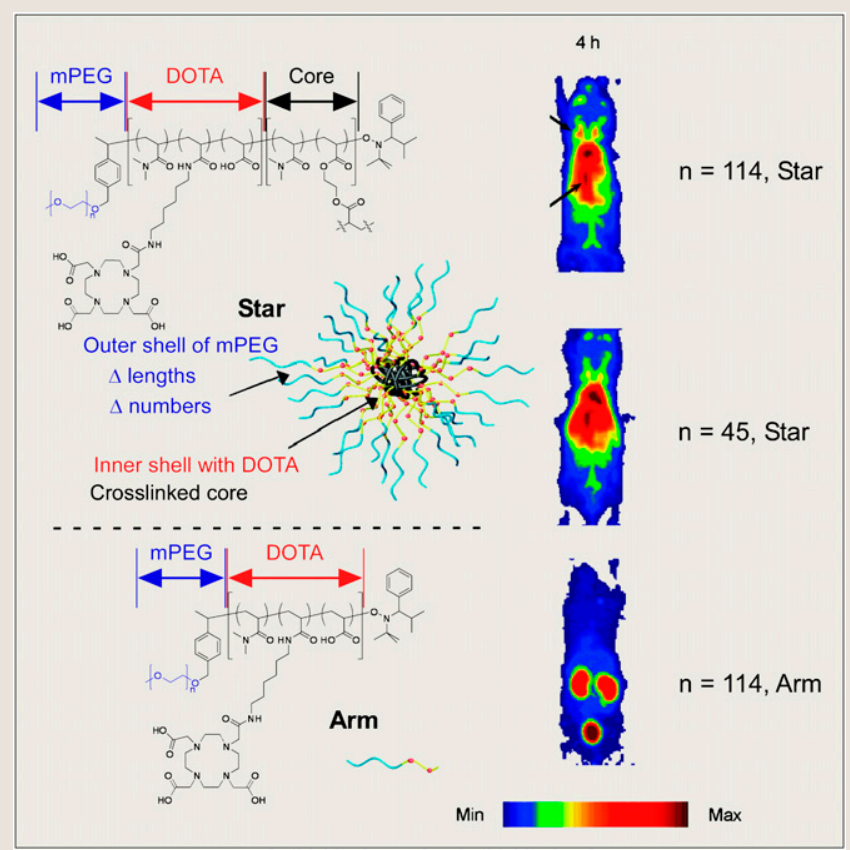

FIGURE 1. Chemical compositions and schematic illustrations of 2 star polymer nanostructures and 1-arm polymer precursor (left side), and small-animal PET projection images (decay-corrected and scaled by minimum-maximum frame) obtained $4 \mathrm{~h}$ after their ${ }^{64} \mathrm{Cu}$ labeling and administration in BALB/C mice ( $\sim 1.5-2 \mathrm{mg} / \mathrm{kg}$ of mouse body weight; $\sim 3.7$ $\mathrm{MBq} /$ mouse) (right side). Arrows point to carotid artery (upper) and aorta (lower).

The length of the mPEG presented from the surfaces of the star polymer-based nanostructures played a significant role in their blood circulation lifetimes, to a greater extent than did the nanoparticle size (over the range of $D_{h}, 25-70 \mathrm{~nm}$ ) or core composition. The blood retention was higher and the hepatic and splenic accumulations were lower for star polymers having 5-kDa mPEG arms $(n=114$, star of Fig. $1 ; D_{h}, 70.4 \pm 8.8 \mathrm{~nm}$ ) than for those derived from 2-kDa mPEG arms $\left(n=45\right.$, star of Fig. $\left.1, \mathrm{D}_{\mathrm{h}}, 67.9 \pm 8.0 \mathrm{~nm}\right)$, as seen by the higher proportion of radioactivity in the heart (blood pool) observed by small-animal PET in BALB/C mice (Fig. 1).

This structural effect was also observed for different macromolecular architectures in which the effect of the mPEG chain length was again shown to have a dramatic effect on the in vivo biodistribution of comblike block graft copolymer nanostructures (3). The comb polymers were prepared by a highly efficient 1 -step copolymerization of small molecule monomers that were designed to impart hydrophobic character, small molecule monomers that carried reactive functionalities for later introduction of DOTA macrocyclic chelators (and other active units), and mPEGylated macromonomers. The comb polymers were amphiphilic and underwent self-assembly in water to produce well-defined core-shell nanoparticles, as visualized by cryo-transmission electron microscopy (cryo-TEM). The $\mathrm{D}_{\mathrm{h}}$ value increased from $9.7 \pm 1.1$ to $17 \pm 2$ to $20 \pm 3 \mathrm{~nm}$ with increasing mPEG chain lengths from 1.1 to 2.0 to $5.0 \mathrm{kDa}$, respectively. Although the $D_{h}$ increased and the number of $m P E G$ grafts per comb polymer decreased with increasing $\mathrm{mPEG}$ chain length, the blood clearance time increased and the hepatic accumulation decreased. At $4 \mathrm{~h}, 60 \%-65 \%$ of the administered 2- and 5-kDa mPEG comb-based nanostructures remained in the blood, and $31 \%$ of the $5-\mathrm{kDa} \mathrm{mPEG}$ comb nanostructures were still circulating at $48 \mathrm{~h}$. In contrast, the 1.1$\mathrm{kDa} \mathrm{mPEG}$ chains were found to be insufficient to provide protection against MPS clearance. This study is an important demonstration of the impact that can be made by the nature of the components (i.e., the length of the mPEG) used in the construction of nanoscale systems.

Additionally important is the relative amount of the building blocks, which are incorporated within the nanoscale framework. In particular, the density of mPEG chains presented from the surface of nanostructures is critical to the ability of the mPEG to shield the nanostructure from protein adsorption and, thereby, inhibit the opsonization process that leads to MPS clearance.

The quantification of the impact of mPEG grafting density on the surface of organic polymer nanoparticles was made through a series of rigorous experimental studies (4). The key to the synthesis of the nanoparticles with well-defined and variable levels of $\mathrm{mPEG}$ surface grafts was the incorporation of the mPEGs onto the backbone of polymer building blocks that were designed to then undergo selfassembly to establish the nanoscopic materials. As illustrated in Figure 2, grafting of mPEG chains onto a poly(acrylic acid) chain segment of a diblock copolymer of poly(acrylic acid) and polystyrene allowed for the number of mPEGs per polymer to be varied. In this study, there were 60 repeat units of each of the diblock copolymer components, and $0,1,2.5$, and 5.5 mPEGs were grafted through consumption of $0 \%, 1.67 \%, 4.17 \%$, and $9.17 \%$, respectively, of the acrylic acid residues. Additionally, approximately 2 DOTAs were coupled to each polymer, because this method had been optimized to achieve high radiolabeling yields for the final nanoparticles. The mPEG-grafted and DOTA-functionalized block copolymers were then assembled into micelles in water, by virtue of their amphiphilic character, and the nanoscopic micellar assemblies were stabilized by the introduction of covalent cross-links throughout the shell layer,

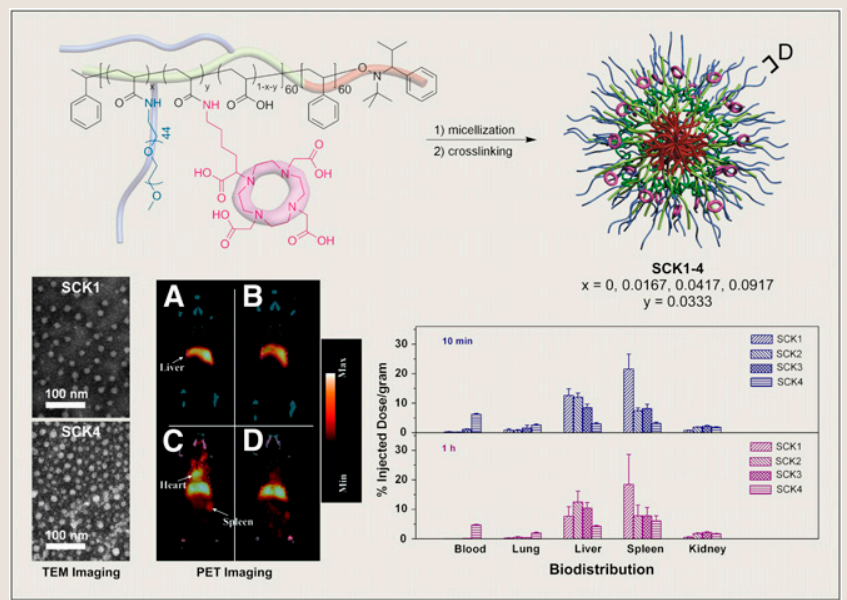

FIGURE 2. (Top) Synthetic approach for quantitative control over mPEG grafting and DOTA loading within SCK nanoparticles. (Lower left) TEM images of 2 SCKs having 0 (SCK1) and 5.5 (SCK4) mPEGs per polymer chain (0 and 1,100 mPEGs, respectively, per SCK nanoparticle). (Lower middle) Coregistered PET/CT coronal images of BALB/C mice injected with ${ }^{64} \mathrm{Cu}$-labeled SCKs $(\sim 3,700-7,400 \mathrm{kBq}$ [100-200 $\mu \mathrm{Ci}$ ] in $150 \mu \mathrm{L}$ via tail vein $[0.15-0.3 \mathrm{mg} / \mathrm{kg}$ of mouse body weight]). Shown are SCK1 at $4 \mathrm{~h}(\mathrm{~A})$ and $24 \mathrm{~h}$ (B) and SCK4 at $4 \mathrm{~h}(\mathrm{C})$ and $24 \mathrm{~h}$ (D). (Lower right) Biodistribution data for ${ }^{64} \mathrm{Cu}$-labeled SCK1-4 in SpragueDawley rats ( $\sim 25 \mathrm{~g} / \mathrm{kg}$ of rat body weight) at $10 \mathrm{~min}$ and $1 \mathrm{~h}$ after injection. 
using a fraction $(20 \%)$ of the residual acrylic acid groups. For this series of shell cross-linked knedellike (SCK) nanoparticles, their $D_{h}$ values were approximately $20 \mathrm{~nm}$, their diameters in the dry state were approximately $15 \mathrm{~nm}$, as measured by TEM, and the spacing between surface mPEG grafts (Fig. 2D) was $2.3 \pm 0.2,1.5 \pm 0.1$, and $1.1 \pm 0.1 \mathrm{~nm}$ for the SCKs originating from the block copolymers having 1.0, 2.5, and 5.5 mPEG grafts, respectively, per polymer.

In vivo small-animal PET in mice and biodistribution in rats indicated that the mPEG surface-grafting densities allowed for tuning of the nanoparticle circulation and deposition profiles. Radiolabeling with ${ }^{64} \mathrm{Cu}$ achieved high specific activities $(>3,700$ $\mathrm{kBq} / \mu \mathrm{g}[>100 \mu \mathrm{Ci} / \mu \mathrm{g}]$ of polymer), which enabled the administration of low doses to healthy rodents $(\sim 5 \mu \mathrm{g} / \mathrm{animal})$. At the highest mPEG surface coverage, for SCK4, having approximately 1,100 mPEGs per nanoparticle and a D-spacing of $1.1 \mathrm{~nm}$, high blood retention and relatively low liver and spleen uptakes were observed, as can be seen by the coregistered small-animal PET/ micro-CT images of SCK4, demonstrating significant levels of radioactivity in the heart at 4 and even $24 \mathrm{~h}$ (Fig. 2, lower middle, $\mathrm{C}$ and D). A D-spacing of approximately $1 \mathrm{~nm}$ has been predicted theoretically to inhibit the adsorption of large and small proteins, thereby inhibiting opsonization and MPS clearance $(5,6)$. The biodistribution data confirmed the unique behavior for SCK4 and other similarly dense mPEG-coated SCKs that were part of the study but are not reported here. In contrast, SCK1, which lacks mPEG, was cleared rapidly from the blood with significant hepatic and splenic uptake (Fig. 2, lower middle, A and B, and lower right). At $48 \mathrm{~h}$ after injection, there was up to $75 \%$ less dose in the liver and spleen for SCK4 than for SCK1. Intermediately, mPEGylated SCKs showed transitional biodistribution behaviors (Fig. 2, lower right).

Dendritic positron-emitting nanoprobes $(\sim 12-\mathrm{nm}$ hydrodynamic radius, as measured by dynamic light scattering) have optimized each aspect of composition, structure, and properties to combine, in 1 welldefined, biodegradable nanoscopic framework, radionuclides for PET, PEG for controlled biodistribution, and ligands for tissue-selective targeting. Eight tyrosine residues near the center of the macromolecular structure were used for ${ }^{76} \mathrm{Br}$ radiolabeling, 8 PEG arms of $2.7-\mathrm{kDa}$ provided extended blood circulation, and cyclic arginine-glycineaspartic (RGD) peptides (disulfide cyclized CRGDC) on approximately 5 of the PEG termini were shown to direct binding to $\alpha \mathrm{v} \beta 3$ integrins (7). This integrin is upregulated at sites of angiogenesis, as occurs in ischemic tissues, tumor vasculature, and the like, and therefore is an important biomarker for several forms of injury or disease. The polyvalent presentation of approximately 5 cyclic RGDs results in a significant 50-fold enhancement in binding affinity to $\alpha v \beta 3$ over a single CRGDC, as measured by a plate-based assay in vitro, and a 6-fold increase in $\alpha \mathrm{v} \beta 3$ receptor-mediated endocytosis in comparison to a dendritic nanoprobe lacking the cyclic RGD peptides, as measured in $\alpha \mathrm{v} \beta 3$-positive cells in vitro. Low doses $(\sim 0.1 \mathrm{mg} / \mathrm{kg})$ of the targeted and nontargeted dendritic PET nanoprobes in healthy mice showed slightly higher accumulation of the targeted nanoprobes in the liver, spleen, kidney, and bone. Only the cyclic CRGDC peptide-conjugated nanoprobe underwent a higher accumulation in a mouse hind limb after ligation and removal of a segment of the femoral artery relative to the nonischemic control limb, showing radioactivity in the entire thickness of the ischemic muscle (Fig. 3); there was no difference between the ischemic and the nonischemic limbs for animals administered the nontargeted nanoprobes. This system, therefore, substantiates the application of small-animal PET as a direct and noninvasive measure of angiogenesis in vivo.
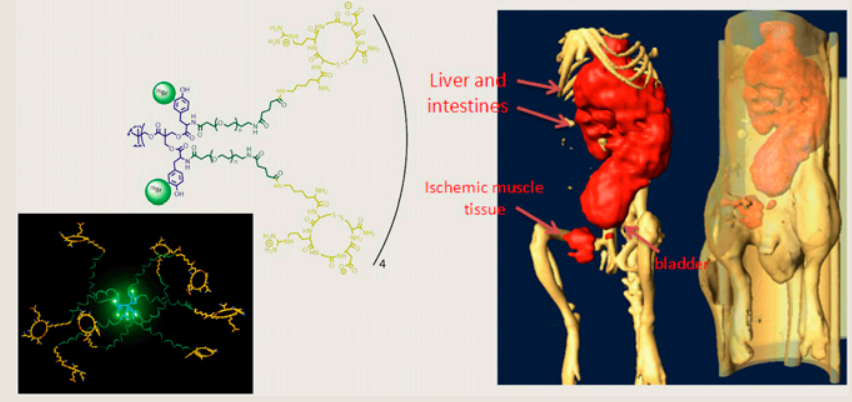

FIGURE 3. Structure of ${ }^{76} \mathrm{Br}$-arginine-glycine-aspartic dendritic positron-emitting nanoprobes targeted to $\alpha \mathrm{v} \beta 3$ integrins and their targeting to angiogenic capillaries in hind limb ischemia mouse model $24 \mathrm{~h}$ after injection.

Control over biodistribution and tissue-selective targeting has also been achieved for inorganic single-walled carbon nanotubes (SWNTs) coated noncovalently with PEG and labeled with cyclic RGD peptides and DOTA for ${ }^{64} \mathrm{Cu}$ chelation and PET (8). PET in vivo demonstrated targeting to $\alpha \mathrm{v} \beta 3$ integrin-positive tumors in mice (Fig. 4) but also significant uptake in the liver. Lower liver and spleen uptakes were observed for SWNTs coated with PEG of $5.4 \mathrm{kDa}$ than for those having shorter, 2-kDa, PEG; the blood halflives were 2 and $0.5 \mathrm{~h}$, respectively. Importantly, although the PEG chains were affixed to the SWNTs noncovalently (via interactions of a phospholipid tail on the PEG with the SWNT surface), rapid renal excretion was not observed, as had been reported earlier for SWNTs $(9,10)$, suggesting that these functionalized SWNTs were quite stable and that the entire nanostructure was trafficking intact in vivo. The SWNTs of this study were more uniform and shorter than typical SWNTs used in engineering materials applications, having lengths of approximately 100-300 nm and diameters of

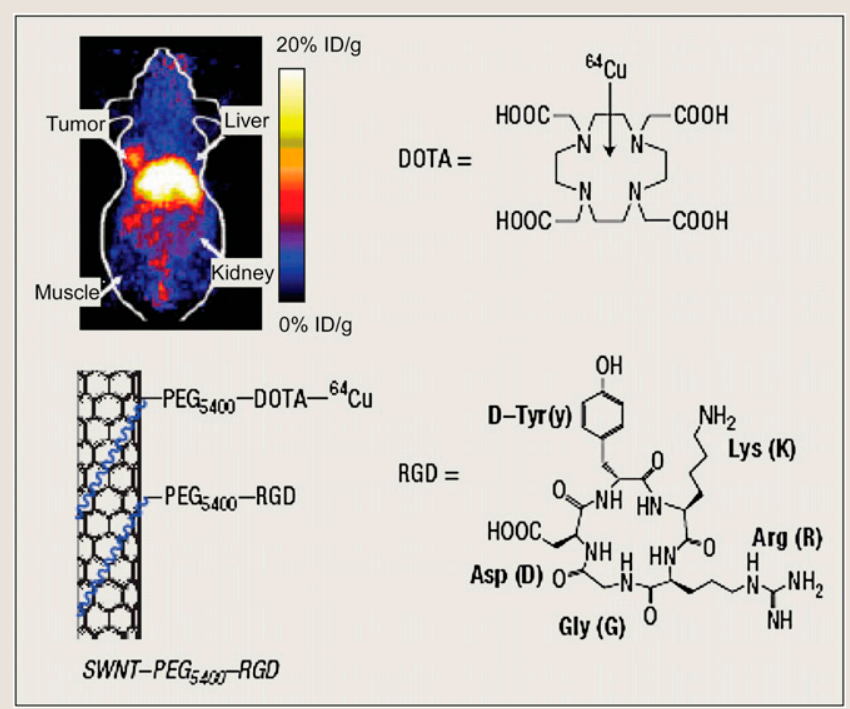

FIGURE 4. Physical adsorption of mixture of phospholipidterminated PEGs having either DOTA or cyclic RGD termini onto SWNTs gave radiolabeled and integrin-targeted rodshaped inorganic nanomaterials that were shown by PET to target $\alpha v \beta 3$-positive tumors in mice. $\% \mathrm{ID} / \mathrm{g}=$ percentage injected dose per gram. 
approximately 1-5 $\mathrm{nm}$. Their size, in combination with their surface chemistry, provided for circulation and tumor localization over time, reaching a plateau of approximately $10-15$ percentage injected dose at approximately $6 \mathrm{~h}$ after injection. The photophysical properties of SWNTs allowed for ex vivo study by Raman spectroscopy, from which the tissue distributions were found to be in agreement with the in vivo PET and ex vivo biodistribution data. The interesting aspects of this work, therefore, include the polyvalent presentation of targeting peptides from a nanoscopic scaffold that allows for multiple radiolabels and imparts additional photophysical characteristics, the rodlike shape of the nanostructures, and the in vivo performance of nanostructures in accomplishing excellent tumor targeting performance. The primary concern with further advances with SWNTs and other inorganic nanomaterials is their mechanism of clearance and implications for long-term accumulation.

Another area of investigation is to develop approaches to increase the number of radionuclides attached to each nanoparticle. An example of using click chemistry to attach large numbers of ${ }^{18} \mathrm{~F}$ atoms has recently been described (11). This approach can use doses of nanoparticles $2-4$ orders of magnitude lower than are required for MRI.

Other advances being made include the development of multimodality agents that can use a single nanoparticle for PET, CT, MR, and optical imaging. Such nanoparticles have been described previously (12). In this example, PET and optical probes are attached to inorganic particles that can also be used for both CT and MRI. In another example (13), targeting ligands were also attached to a magnetic nanoparticle platform allowing both PET and MRI.

One challenge in the application of nanoparticles for oncologic imaging is that target uptake can be achieved using both a targeting ligand and a nonspecific uptake called the enhanced permeability and retention (EPR) effect. If one is using the nanoparticle simply for determining whether a tumor exists, the EPR effect can be a bonus; however, if one wishes to know the amount of uptake due to a targeting ligand, this effect needs to be minimized. The EPR effect of various tumor sizes has been investigated for the folate receptor (14). Approaches to exploit the EPR effects to increase the uptake by this mechanism have been discussed (15), as has the methodology to distinguish between EPR uptake and uptake using a targeting ligand (16).

\section{CONCLUSION}

Nanoparticles show great promise, yet much discovery and development still remains to be done. The examples presented in this review were selected to exemplify the ability to significantly alter the biodistribution of nanostructures by minor structural changes, highlighting the importance of well-defined chemistries for their preparation, in concert with rigorous analytic tools for their physicochemical characterization, and PET for study of their in vivo performance. PET serves as a highly sensitive imaging tool to assist in the development of these materials to realize the potential of nanomedicine for early-stage detection, diagnosis, treatment, and monitoring of disease progression, regression, and recurrence.

The primary challenges over the next $5 \mathrm{y}$ will be to accomplish truly tissue-selective targeting, without the significant MPS organ uptake, and full clearance of the nanomaterials once they have served their purpose. Toxicity and immunogenicity are measured routinely for nearly every new nanomaterial being investigated. The examples presented here included materials that have not displayed adverse biologic responses, and they are also structures that are robust- the robust character was important in the early studies to probe the behavior of the nanostructures while they remained as intact nanoscale objects. However, as development moves forward, it will be important to replace the chemically stable components with others that are biodegradable. In this aspect, again, organic polymer materials have advantages over inorganic substrates, because several families of organic polymers are used regularly with Food and Drug Administration approval for biomedical applications (poly[lactic acid] and poly[glycolic acid], among many others).

\section{ACKNOWLEDGMENT}

This work is supported by the National Heart, Lung and Blood Institute of the National Institutes of Health as a Program of Excellence in Nanotechnology (HL080729).

\section{REFERENCES}

1. Cherry SR. The 2006 Henry N. Wagner lecture: of mice and men (and positrons) - advances in PET imaging technology. J Nucl Med. 2006;47:17351745.

2. Fukukawa K, Rossin R, Hagooly A, et al. Synthesis and characterization of coreshell star copolymers for in vivo PET imaging applications. Biomacromolecules. 2008;9:1329-1339.

3. Pressly ED, Rossin R, Hagooly A, et al. Structural effects on the biodistribution and positron emission tomography (PET) imaging of well-defined $\mathrm{Cu}$-64-labeled nanoparticles comprised of amphiphilic block graft copolymers. Biomacromolecules. 2007;8:3126-3134.

4. Sun G, Hagooly A, Xu J, et al. Facile, efficient approach to accomplish tunable chemistries and variable biodistributions for shell cross-linked nanoparticles. Biomacromolecules. 2008;9:1997-2006.

5. Jeon SI, Andrade JD. Protein-surface interactions in the presence of polyethylene oxide: II. effect of protein size. J Colloid Interface Sci. 1991;142:159-166.

6. Jeon SI, Lee JH, Andrade JD, De Gennes PG. Protein-surface interactions in the presence of polyethylene oxide: I. simplified theory. J Colloid Interface Sci. 1991;142:149-158.

7. Almutairi A, Rossin R, Shokeen M, et al. Biodegradable dendritic positronemitting nanoprobes for the noninvasive imaging of angiogenesis. PNAS. 2009; 106:685-690.

8. Liu Z, Cai W, He L, et al. In vivo biodistribution and highly efficient tumour targeting of carbon nanotubes in mice. Nat Nanotechnol. 2007;2:47-52.

9. Singh R, Pantarotto D, Lacerda L, et al. Tissue biodistribution and blood clearance rates of intravenously administered carbon nanotube radiotracers. Proc Natl Acad Sci USA. 2006;103:3357-3362.

10. Wang H, Wang J, Deng X, et al. Biodistribution of carbon single-wall carbon nanotubes in mice. J Nanosci Nanotechnol. 2004;4:1019-1024.

11. Devaraj NK, Keliher EJ, Thurber GM, Nahrendorf M, Weissleder R. F-18-labeled nanoparticles for in vivo PET-CT imaging. Bioconjug Chem. 2009;20:397-401.

12. Nahrendorf M, Zhang H, Hembrador S, et al. Nanoparticle PET-CT imaging of macrophages in inflammatory atherosclerosis. Circulation. 2008;117:379-387.

13. Lee HY, Li Z, Chen K, et al. PET/MRI dual-modality tumor imaging using arginine-glycine-aspartic (RGD)-conjugated radiolabeled iron oxide nanoparticles. J Nucl Med. 2008;49:1371-1379.

14. Rossin R, Pan D, Kai Q, et al. ${ }^{64} \mathrm{Cu}$-labeled folate conjugated shell cross-linked nanoparticles for tumor imaging and radiotherapy: synthesis, radiolabeling, and biologic evaluation. J Nucl Med. 2005;46:1210-1218.

15. Iyer AK, Khaled G, Fang J, Maeda H. Exploiting the enhanced permeability and retention effect for tumor targeting. Drug Discov Today. 2006;11:812-818.

16. Pirollo KF, Chang EH. Does a targeting ligand influence nanoparticle tumor localization or uptake? Trends Biotechnol. 2008;26:552-558. 